\title{
5 THE POLITICS OF INFORMATION AND COMMUNICATION TECHNOLOGY DIFFUSION: A Case Study in a UK Primary Health Care Trust
}

\author{
David W. Wainwright \\ University of Northumbria \\ Newcastle upon Tyne, UK \\ Teresa S. Waring \\ University of Sunderland \\ Sunderland, UK
}

\begin{abstract}
This paper investigates the politics of ICT diffusion and presents the findings from a pilot study conducted across general medical practices based within a Primary Care Trust in the North East of England. An overview is provided of the macro level politics of ICT adoption in a UK primary health care and the applicability of diffusion of innovation research within the healthcare context. A research approach, based on phenomenology, semi-structured interviews, and template analysis is adopted in the study in order to conduct and provide a rich analysis of the data. The findings are discussed using a modified diffusion of innovation framework. Conclusions highlight how ICT innovation is politically constrained, perceived, and motivated within primary healthcare environments and how in this case it might influence organizational resilience.
\end{abstract}

Keywords Diffusion of innovation, ICT, primary health care, general medical practice, template analysis, organization resilience

\section{INTRODUCTION}

Computer information and communication technology (ICT) entered medical general practice in the UK in 1970 when John Preece became the first British medical general practitioner to use a computer in his consulting room. Five years later, the

\footnotetext{
Please use the following format when citing this chapter:

Wainwright, David, W., Waring, Teresa, S., 2006, in International Federation for Information Processing (IFIP), Volume 206, The Transfer and Diffusion of Information Technology for Organizational Resilience, eds. B. Donnellan, Larsen T., Levine L., DeGross J. (Boston: Springer), pp. 71-90.
} 
health center at Ottery St Mary, near Exeter, was the world's first paperless general practice. During the 1980s, GPs could participate in the "Micros for GPs" scheme where subsidized technology was supplied by the government to encourage innovation. This was followed by two general practice ICT suppliers offering free computer schemes which eventually covered 20 percent of all English GP practices. After a period of stagnation in the 1990s, most GPs are now connected to the NHS net, the internal National Health Service (NHS) network, and many are using e-mail and the Internet (Benson 2002). Thus there has been a 35 year period where ICT in general practice has had the potential to grow, develop in sophistication, support innovation, and become normalized throughout the GP community. Nevertheless, despite numerous versions of the UK government's NHS IT strategy, and various funding initiatives, this has yet to happen. The future organizational resilience of the NHS in general, and primary care in particular, is a growing concern, especially in relation to the new and massive investment in ICT innovations. Successive reforms of the healtheare system are becoming increasingly dependent on efficiency gains achieved through organizational change, the modernization of professional work practices, and the increasingly ambitious levels of ICT adoption. Organizational resilience within the healthcare sector has become integrally linked with modernization that, in turn, depends on effective ICT adoption practices.

In this context, a useful definition of organizational resilience is provided by Riolli and Savicki (2003, p. 227) who adopt the definition as provided by Horne and Orr (1998):

Resilience is the fundamental quality of individuals, groups, organizations, and systems as a whole to respond productively to significant change that disrupts the expected pattern of events without engaging in an extended period of regressive behavior.

They particularly focus on factors related to information systems adoption with respect to vulnerabilities and stress at both the level of the individual and the organization.

This paper investigates the politics of ICT diffusion and presents the findings from a pilot study conducted with GP practices in a Primary Care Trust in the North East of England. In the context of our research general medical practices in the UK can be viewed as small businesses (GPs are self employed and have a contractual arrangement with the English NHS). The first section provides an overview of the macro level politics of ICT adoption in UK primary health care and then, in the second section, goes on to consider diffusion of innovation (DOI) research and its applicability in the healthcare context. The third section explores the research methodology, the template analysis adopted in the study, and the findings that emerged from the large amount of rich data collected through semi-structured interviews. The final section, comprising discussion and conclusions, highlights the findings in relation to how the process of diffusion of innovation, in this case healthcare ICT, might determine future organizational resilience together with a reflection on the approach chosen and its appropriateness to the study. 


\section{ICT AND THE MACRO POLITICS OF THE NHS}

The ICT strategy of the English NHS has a long and checkered history - not least because it changes its objectives, scope, and emphasis at very frequent intervals. This mirrors the dominant government political ideologies (for a more detailed account see Wainwright and Waring 2000). The strategy, throughout the 1990s and up until the present, has evolved from a focus on resource management, integrated hospital information support systems (HISS), the electronic patient record (EPR), to what is now a lifetime "cradle to grave" record termed the electronic health record (EHR). Lately, after the millennium, a major step change in ICT investment has taken place in parallel with government policies for modernization of the entire health service organization. There are official estimates of $£ 6.5$ billion and unofficial, but not really disputed, forecasts of up to $£ 30$ billion of investment in ICT hardware, software, consultancy services, training, and infrastructure over an initial 8 year period with a fully integrated ICT enabled health service targeted for 2010. This is governed under the organizational umbrella of the National Programme for Information Technology (NPfIT, www.npfit.nhs.uk), initiated in October 2002. In 2005 this became known as Connecting for Health (DOH 2005), a government agency, which is tasked with developing and implementing a new care records service (CRS) to connect secondary care hospitals with primary care healthcare providers (general medical practices).

There is a paucity of research that examines ICT adoption within both the UK NHS generally (Wainwright and Waring 2000) and primary care services specifically (Newton et al. 2003). The politics of diffusion of ICT, and the impact of managerial instrumentalism within a professional service culture, has been the focus of a small number of interpretive and critical theoretical studies over the past decade (for example, Bloomfield et al. 1997; Doherty, Coombs, and Loan-Clark 2004; Doherty, King, andMarples 2000; Packwood et al. 1998; Pettigrew et al. 1994; Waring and Wainwright 2002). These studies have examined how political agendas have been the driving force for a process of modernization or significant healthcare reforms - often viewed as the rise of managerialism, centralized control, and an assault on, or an erosion of, power of the medical profession. The NHS itself has kept a very tight control on the dissemination of information about the progress of the national ICT strategy. This has caused problems for many independent researchers who have requested access to the NHS over the last 8 years.

\section{DIFFUSION OF INNOVATION RESEARCH AND ITS APPLICABILITY TO HEALTHCARE}

Diffusion of innovation research and practice originates from many diverse fields of study. These include sociology, anthropology, healthcare, medicine, social policy, psychology, strategic management, economics, marketing, entrepreneurship, organizational behavior, research and development, and technology management. An innovation is not just an outcome but a process and is the effort to create purposeful, focused change in an enterprise's economic or social potential (Drucker 1985). It may be viewed as something that is new to an adopting organization but not necessarily new in its own 
right. Rogers (1995) over the course of many decades has developed and refined a diffusion of innovation (DOI) framework. He defines diffusion as the process by which an innovation is communicated through certain channels over time among the members of a social system and that an innovation is an idea, practice, or object perceived as new by an individual or other unit of adoption. Also, that there are five attributes of innovation: relative advantage, compatibility, complexity, trialability, and observability. Similarly, five steps are involved in the innovation decision process: knowledge, persuasion, decision, implementation, and confirmation. Five adopter categories based on innovativeness are identified: innovators, adopters, early majority, late majority, and laggards, where the rate of adoption is the relative speed at which an innovation is adopted by members of a social system. The social and communication structure of a system is seen to either facilitate or impede diffusion where norms, opinion leadership, change agentry, and aides are seen as key variables in the process. Finally, four types of innovation decisions are identified: optional (independent choices), collective (consensus), authority (power enforced by a few members), and contingent (choices made after a prior decision). The main corpus of work related to DOI studies provides useful generic theories, based on the innovation definitions and processes identified, to explain technology adoption in many diverse fields. Most of this work relates to studies of large organizations. However, there is a growing interest in research on IT adoption and e-business specifically in small to medium, private sector businesses (Caldeira and Ward 2003; Cragg 2002; Grandon and Pearson 2004; Poon and Swatman 1999; Southern and Tilley 2000). These studies tend to adopt a case study approach, building competing theories of ICT adoption but not specifically utilizing any DOI framework. More recent studies and particularly those in the area of Information Systems research, attempt to explain ICT adoption and use by building on and extending the classic Rogers DOI framework. These adapted theories may, therefore, be more applicable to aid understanding and knowledge related to ICT adoption in healthcare environments.

\subsection{Diffusion of Innovation Research and Information Systems}

It is apparent that in the area of Information Systems there is a growing body of academic literature related to diffusion of innovation theory and that this ranges from macro-industry level studies of Internet adoption through to organizational and microlevel individual adoption of technologies such as software process improvements (Mustonen-Ollila and Lyytinen 2003). Kim and Galliers (2004) provide a comprehensive review of diffusion studies related to IS and also provide a model to explain the diffusion of Internet systems comprising four dimensions: external market factors, external technical factors, internal organizational factors, and internal systems factors. Although useful for broadly explaining the diffusion of ICT and Internet systems at the macro (industry) and micro (organizational and individual) levels the model does not provide an adequate theoretical basis for exploring more complex diffusion processes associated with organizations and their associated power and political dimensions. Other studies, however, attempt to more directly examine information systems process innovations and retrospectively apply them to primary longitudinal case data utilizing DOI theory. 
One such study by Kautz and Larsen (2000) utilizes and adapts the original DOI framework of Rogers. They use this to examine the diffusion of software process innovations by utilizing some key elements of the DOI framework to revisit and reanalyze the findings from the European Software Process Improvement Training Initiative (ESPITI) in Norway. A key aim of this research was to discuss the suitability and limitations of Roger's framework as retrospectively applied to this project. The case analysis adopted categories of innovation content and context, information needs and target audience, change agents and opinion leaders, and finally innovation consequences and countermeasures. Kautz and Larsen conclude that the conscious use of Rogers' DOI framework might have resulted in a better dissemination strategy and might have been beneficial for the project. A criticism, however, was that the model does not adequately address the dynamic relationship between all stakeholders involved in the ESPITI project: the "classic" diffusion process, often being perceived as too instrumental, driven by the needs of potential adopters and also as overly sequential in nature.

A second study by Baskerville and Pries-Heje (2001) utilizes three innovation diffusion models: the interactive model, the linked-chain model, and the emergent model to produce distinctive but complementary knowledge (a retrospective analysis using competing theories) about three information technology case settings. Importantly, they describe the diffusion of innovation process in terms of an "ecological" view (centralizes conflict and competition in the diffusion setting) and a "genealogical" view (centralizes consensus and regulation). This is where the micro perspective is a genealogical view based on economics and innovation theory and the meso and macro perspectives are ecological views that use power dependency analysis on networks of interacting agencies to understand how extra-organizational power dependencies shape the diffusion process (Baskerville and Pries-Heje (2001, p. 182). The classic innovation literature is seen to typically adopt the genealogical view. Innovation is, therefore, seen as a linear process, either as technology push, (market/adopter) need-pull, or interactive (both technology linking and need linking). The linked chain model is also positioned within the genealogical view but incorporates a knowledge dimension as the base of the innovation and its diffusion. Emergent or ecological models of innovation are based on conflict theories where innovation may be stimulated by internal or external shocks to the organization: unpredictable setbacks and surprises are inevitable and restructuring of the organization often occurs.

So, like a social culture, innovation evolves as mixture of old and new ideas, directions and stakeholders; outcomes, evaluation and histories of events are "reframed," contemporaneously conflated or partitioned in order to rationalize "the messy and complex progression of ideas observed in the innovation cases" (Baskerville and Pries-Heje 2001, p. 187, quoting Schroeder et al. 1989).

Interestingly, each DOI theory utilized proved equally meritorious and provided complementary and distinctive knowledge; there was no one best model. In terms of advice to other researchers, therefore, the ecological view is recommended in research focused on multiple entities involving evolution or confrontation and dialectic. This is certainly more characteristic of healthcare environments, which exhibit multiple organization structures, have a long history of power struggles between professional groupings, and are shaped by a very intense political macro environment. 
These studies present important findings in relation to the application of DOI theoretical frameworks to explain complex organizational scenarios for information systems process innovations. In order to perform a more detailed case analysis, however, a more structured framework would provide advantages in terms of developing or complementing an analytical template to explain and understand ICT diffusion studies. More recently, Mustonen-Ollila and Lyttinen (2003) attempt to develop such a framework in order to undertake a longitudinal analysis of process innovations. They expand, adopt, and adapt Rogers' DOI theory and the original list of five factors that affect innovation adoption: innovation, individual, task, environmental, and organizational. Based on these original categories, and from a review of relevant IS diffusion studies literature, 28 attributes are identified that represent independent variables; the dependent variable being the likelihood to adopt an innovation. This can be viewed in Table 1.

Table 1. Diffusion of Innovation Factors (adapted from Mustonen-Ollila and Lyytinen 2003 , p. 282).

\begin{tabular}{|c|c|c|}
\hline $\begin{array}{l}\text { DOI } \\
\text { Factor }\end{array}$ & Characteristic & Explanation \\
\hline 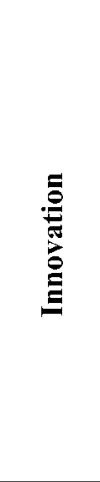 & $\begin{array}{l}\text { - Relative advantage } \\
\text { - Ease of use } \\
\text { - Compatibility } \\
\text { - Visibility } \\
\text { - Trialability } \\
\text { - Price } \\
\text { - Problem solver } \\
\text { - Standard } \\
\text { - Technological edge }\end{array}$ & $\begin{array}{l}\text { - It is thought better than the idea it replaces. } \\
\text { - Is it difficult to use or understand. } \\
\text { - The degree to which an innovation is perceived } \\
\text { consistent with the existing values, past experiences } \\
\text { and the needs of potential adopters, similar to } \\
\text { suitability. } \\
\text { - Is it experimental. } \\
\text { - Is the cost of the innovation excessive. } \\
\text { - Does the innovation solve a problem not currently } \\
\text { dealt with. } \\
\text { - The innovation becomes the standard in the sector } \\
\text { and clients are forced to use. } \\
\text { - Superiority to other innovations. }\end{array}$ \\
\hline th & $\begin{array}{l}\text { - Commercial advantage } \\
\text { - User need recognition } \\
\text { - User resistance }\end{array}$ & $\begin{array}{l}\text { - The internal or external vendor sells an innovation in } \\
\text { a form of a useful product. Later on this product is } \\
\text { commercialized. } \\
\text { - An innovation must match the user needs in the task. } \\
\text { - Innovations must simplify tasks to avoid resistance. }\end{array}$ \\
\hline ב & $\begin{array}{l}\text { - Own testing } \\
\text { - Personal contact network } \\
\text { - Own rules and control of } \\
\text { own work } \\
\text { - Learning by doing }\end{array}$ & $\begin{array}{l}\text { - Innovation used experimentally by individual. } \\
\text { - People use their own networks to disseminate } \\
\text { innovation. } \\
\text { - The innovator has to conduct experimentation with } \\
\text { the new idea in order to assure itself that innovation } \\
\text { is advantageous. } \\
\text { - Learning to evaluate the innovations on the basis of } \\
\text { experience. }\end{array}$ \\
\hline
\end{tabular}




\begin{tabular}{|c|c|c|}
\hline $\begin{array}{c}\text { DOI } \\
\text { Factor }\end{array}$ & Characteristic & Explanation \\
\hline 产 & $\begin{array}{l}\text { - Cultural values } \\
\text { - Technological } \\
\text { infrastructure } \\
\text { - Community norms } \\
\text { - Funding }\end{array}$ & $\begin{array}{l}\text { - Cultural beliefs concerning change. } \\
\text { - The maturity of the technological infrastructure. } \\
\text { - To what extent will all conform. } \\
\text { - Are resources available to help in the diffusion }\end{array}$ \\
\hline : & $\begin{array}{l}\text { - Interpersonal networks } \\
\text { - Peer networks } \\
\text { - Informal communication } \\
\text { - Technological } \\
\text { experience } \\
\text { - Working teams } \\
\text { - Opinion leaders and } \\
\text { change agents } \\
\text { - Interdependence from } \\
\text { others } \\
\text { - Adopter type } \\
\text { - Management hierarchy }\end{array}$ & $\begin{array}{l}\text { - Evaluations of innovations are shared between } \\
\text { individuals. } \\
\text { - Social relationships. } \\
\text { - Exchange of information is informal and unplanned. } \\
\text { - Technological experience over a long period of } \\
\text { time-good or bad. } \\
\text { - Team members keeping primary control over their } \\
\text { management. } \\
\text { - An individual who influences clients' innovation } \\
\text { decisions-are they present? } \\
\text { - Each adopter increases the utility of the innovation } \\
\text { for both future adopters. } \\
\text { - The degree to which an individual is earlier in } \\
\text { adopting new ideas than others. } \\
\text { - Do management determine the adoption of an } \\
\text { innovation. }\end{array}$ \\
\hline
\end{tabular}

\subsection{Selecting a Diffusion of Innovation Research Model to Explain ICT Adoption in Healthcare}

The diffusion, spread, and sustainability of ICT innovations within health service delivery and organization may be examined from multiple perspectives of innovation in terms of organization structure, work processes, professional cultures, services delivery, clinical working, products, processes, and technologies. After a review of relevant literature, it appears that many competing DOI theories (Baskerville and PriesHeje 2001) could be used; each would provide an interesting and a potentially complementary account of our research project. However, in the spirit of our particular pilot study, which was exploratory in nature, it would seem prudent to commence by using elements from the classic DOI framework as articulated by Rogers. Also, as the research was focused on determining diffusion behaviors, politics, and processes across a complex social network (healthcare) there was a need to utilize a framework that more clearly articulated a range of relevant factors mapped to Rogers' DOI theory. Having explored a number of adaptations of DOI theory, the framework developed by Mustonen-Ollila and Lyttinen, in their study of information system process innovations, was, from our perspective, the most comprehensive and suitable structure upon which to base our analysis. It also provided the possibility to incorporate elements related both the genealogical and also ecological views (Baskerville and Pries-Heje 2001), as innovation occurs at different levels of resolution. Therefore, issues related to the 
individual, as well as the social unit and the enterprise system, should be examined. Our focus is on the politics of ICT diffusion but also incorporates a much broader view of the concept of innovation and diffusion within complex social networks. The next section deals with the primary research and the methodology used.

\section{THE RESEARCH METHODOLOGY}

The methodology that we adopted in this research was that of phenomenology based on template analysis. According to King (2004, p. 256),

The essence of template analysis is that the researcher produces a list of codes representing themes identified in their textual data. Some of these will usually be identified a priori, they will be modified and added to as the researcher reads and interprets the texts.

The approach may be considered sympathetic to grounded theory but is not as prescriptive in terms of rigorously following a purely inductive pathway. Its advantages include the ability to develop conceptual themes and the clustering into broader groupings with the eventual identification across cases of master themes. Template analysis works particularly well when the aim is to compare the perspectives of different groups of staff within a specific context (King 2004, p. 257).

\subsection{The Study: The Use of ICT in Primary Care}

The project described here explores the impact of NHS information and communication technology (ICT) initiatives at the level of general practice organizations. There is little empirical research exploring current issues concerning ICT strategy adoption and usage within primary care from the user as opposed to policy maker perspective. We wanted to define key issues impacting upon the adoption of new ICT within primary care practice from clinician, management, and administrative stakeholder perspectives. We then aimed to evaluate the progress of ICT adoption and its diffusion relative to targets defined within the current NHS information strategy. The research was designed and carried out by the authors. Two main research questions were posed.

- To what extent has the current NHS ICT strategy and diffusion of ICT impacted on working lives at the level of the general medical practice?

- What are general medical practices' experiences of engaging in ICT initiatives and how will this affect future adoption of ICT within primary care organizations?

Initially we approached Gateshead Primary Care Trust (PCT), in the North of England, to explore whether we could carry out the research with a number of their GP practices. The PCT was a relatively new organization, created under the most recent wave of NHS and labor government organizational reforms. The staff in the PCT were only beginning to establish an understanding of the ICT issues within the Trust. The 
senior management of the PCT were very supportive of the research and we were asked to submit our research proposal to both the Gateshead PCT ethics and research and development committees. Eventually, after almost 12 months and further revisions, we were given clearance to carry out the research (this is not unusual in English health services research).

Initially a pilot site was selected for the first trial run of the data collection. This enabled the research design to be reviewed, assessed, and refined prior to expanding it across a wider range of respondents and organizations. The study was conducted between January and September 2004 and aided by a small university research grant.

In discussion with the PCT, we thought that we would like the study to include GP practices that fit the following profile in order to get a representative cross-section across the PCT:

- Opportunity, willingness and potential for good collaboration

- Mix of local general medical practices

- Levels of ICT usage and maturity (paper-heavy to paper-lite)

- Involvement in specific ICT projects, electronic health records, Prodigy (decision support systems for doctors and nurses), pathology and laboratory results reporting, hospital booked patient admissions, etc.

However, after some discussion we agreed with the PCT that GP practices should be sent a letter by the PCT inviting them to participate in the pilot research project. From the responses to these letters, five practices were identified. Obviously these practices were self-selecting and this poses issues for the validity of the study and the findings that have emerged. However, this was the best situation available and it may not reflect the PCT as a whole. The method of data collection for this study was that of the semistructured interview. At each of the five research sites, tape recorded interviews were carried out with a core set of staff: a general practitioner (doctor), practice manager, and practice nurse. The interviews were based around a broad set of topic headings to guide the interviews but we tried as far as possible to allow the participants to lead the discussion. Research consent forms and information leaflets were provided in advance of the study.

Interviews took place at a time and place convenient to the respondent and ideally were limited to a maximum of 1 hour duration. All data has been made anonymous and all confidences respected. Transcripts (verbatim) were presented back for verification by each respondent. Wherever possible, the two researchers were present to enable consistency of the data collection approach and to enable peer reflexivity at a later date in terms of the data analysis and also the research process itself.

\subsection{Developing the Template}

In this section we will describe the development of the analytical template but it is essential to recognize that the development of the template is not a separate stage from its usage in the analysis of texts. 
Defining the codes and creating the initial template: Template analysis normally starts with some predefined codes intended to help guide analysis. The first issue we faced was how extensive the initial template should be. King (2004) suggests that if you start with too many predefined codes, then the template might blinker analysis and prevent exploration of more pertinent issues. On the other hand, too few codes may lead to an overwhelming mass of rich and complex data.

Our approach and starting point was the letter sent out to the GP practices introducing the research topic and some of the government and department of health concerns. From these we constructed a template in a graphical format (see Figure 1). This took a period of time and reflection to ensure we agreed on the initial codes. The template was used as a prompt for the respondent and adapted for use if necessary. The key words have been derived from the research literature, NHS documentation related to ICT strategy initiatives, and the personal research experience of the researchers. This approach has a proven record of successful use in qualitative research in healthcare settings specifically investigating "soft" organizational issues (Clarke and Wilcockson 2001, 2002).

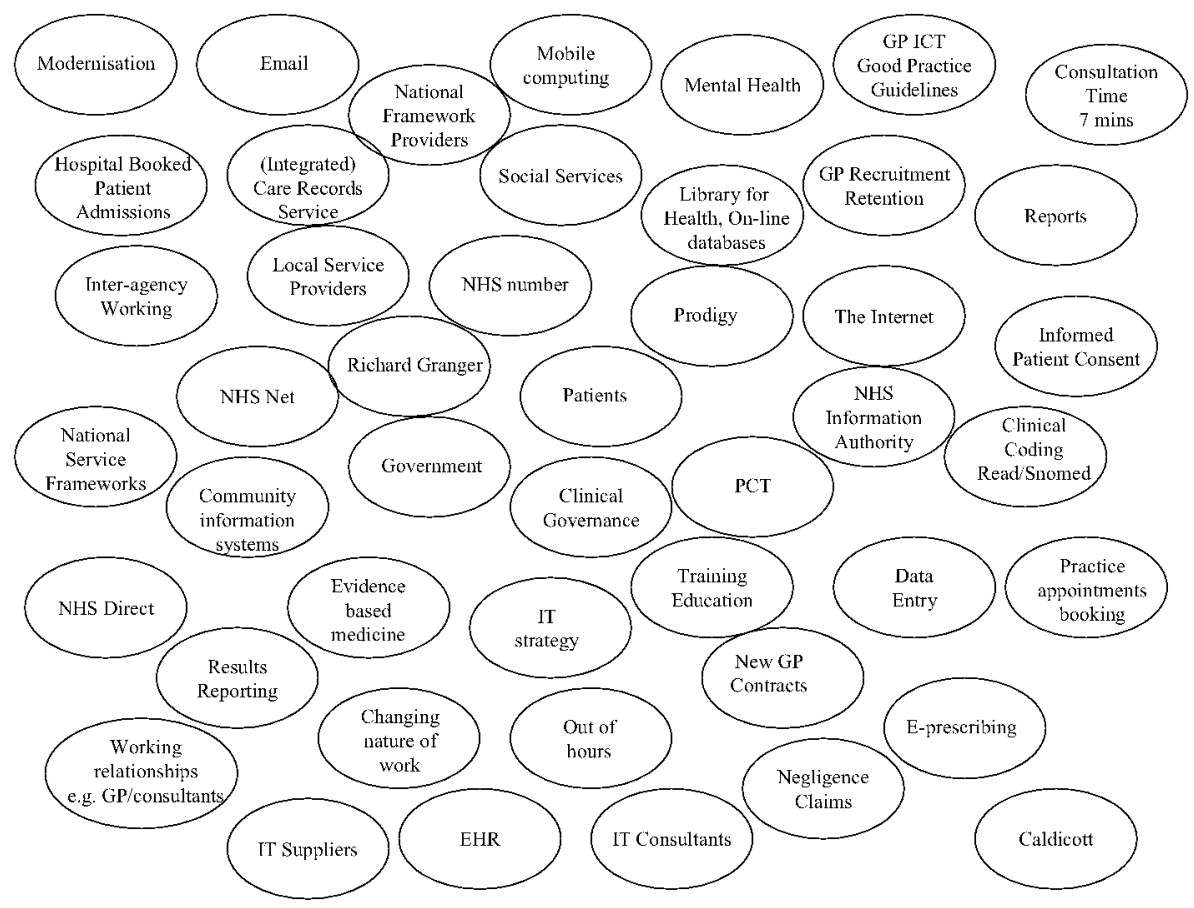

Figure 1. Template for Key Issues Associated with ICT in English General Medical Practices 
Revising the template: It is important to realize that the template must be revised in response to the concerns of the interviewees. Respondents were able to suggest other key words that they thought should be included and enlarged on them if they felt it necessary. They were provided with the template at least 1 week prior to the interview. As part of the research we soon discovered that many of the key issues, words, and phrases that we identified initially were not relevant to all of the interviewees and a number could be deleted. It was also apparent that GPs had issues and perspectives different from nurses and practice managers even when they came from the same organization practice.

\section{FINDINGS}

In order to analyze our results we have used the diffusion of innovation factors as developed by Rogers (1995) and have addressed, to a greater extent, the attributes of these factors as set out by Mustonen-Ollila and Lyytinen (2003). Unfortunately, the large amount of rich data collected in this study is difficult to encapsulate in such a short paper and so we have developed a framework as shown in Table 2 that highlights the factors but also incorporates political issues that are intrinsically linked to the diffusion agenda within the study.

If we now consider each section in turn we will be able to highlight some of the key issues that have emerged and determine the extent to which policy and reality are mirrored at the level of the GP practice.

Innovation: The term innovation has connotations with leading-edge technology and new and better ways of doing tasks. Yet Rogers recognized that innovation can have many meanings in different stakeholder contexts. In the case of Gateshead PCT, and the pilot practices therein, innovation means optimizing the use of EMIS (this is the medical practice computer system used by all the practices sampled and also by 55 percent of doctors in England; see emis-online.com). EMIS is constantly evolving and has many features, a number of which have not previously been used by the staff in the practices. However, the new environment of the GP contract (a new NHS government reform related to the adoption of new working practices for doctors allied to significantly enhanced financial incentives) means they have to engage more with the data to meet targets, demonstrate quality, and enable financial probity. There is also the ambition of some practices, driven by PCT "e-rhetoric," to implement full electronic patient records systems and become "paper-lite." Nevertheless, when this was explored further, it was difficult to establish to what extent staff in a practice understood these terms.

One interesting aspect of the PCT was the use of mobile technology. Community nurses based centrally at the PCT headquarters used notebook computers with wireless technology when visiting patients' homes. Instead of supporting the work of the GPs, this was a cause for some concern as practices were not consistent in their data entry requirements and community nurses appeared to have a limited appreciation of their obligations. Staff at the practices often had to re-input data entries for consistency. Only one of the doctors we interviewed had used mobile technology (a type of Palm Pilot) while visiting patients and it had limited appeal: 
Table 2. Diffusion of Innovation Factors Applied to the Political Issues of the Process in the GP Research Sites ${ }^{1}$

\begin{tabular}{|l|l|l|l|}
\hline \multicolumn{1}{|c|}{ GPs (Doctors) } & \multicolumn{1}{|c|}{ Nurses } & Practice Managers \\
\hline & Mobile technology & - Internet & - Paper-lite systems \\
\hline & New use of EMIS- & Results reporting & - E-communications \\
Population Manager & - EMIS - Population & - EMIS Population \\
- Manager
\end{tabular}

${ }^{1}$ Table 2 shows contextualized attributes for key stakeholders in GP practices, adapted from "Why Organizations Adopt Information System Process Innovations: A Longitudinal Study Using Diffusion of Innovation Theory," E. Mustonen-Ollila and K. Lyytinen, Information Systems Journal (13), 2003, p. 282. 


\section{I decided to review the medication of everybody over 75 , a certain percentage of which were housebound. They gave me a Palm Pilot, it was too cumber- some...we've printed off some forms.... we all know how to use that.... it's a lot easier.}

Taking hard copy out to patients was far more efficient in their eyes. This was also reinforced by new NHS structural reforms that enabled unsocial "out of hours" services to be outsourced to a collective of locum tenentes GPs. This resulted in GPs doing for fewer home visits to patients.

The Internet is becoming a resource that is encroaching on GP practices in varying degrees. The GPs saw it as a useful resource for information but did not use it extensively in consultations. However, the nurse practitioners found it extremely helpful when dealing with patients and often used it to support the management of illness.

A final innovation in the pilot practices was the recent ability to receive results of patients' tests (blood, etc.) directly into the surgery from the local hospital via e-mail. This is not at the forefront of technological innovation but for these individuals it has been one of the major developments in supporting their work and when it malfunctions they are distraught. One nurse commented:

We didn't get any results! We got no results for a long time, we couldn't download it... we had to go back to paper copies.

Task: Although ICT has been around for many years and GPs have used it to a greater or lesser extent, it is now evident that it is becoming ubiquitous in these pilot practices. The increasing dependence is illustrated by one GP:

\section{Recently we've become paper-lite so we're now so dependent on the computer and we use it so frequently it's integral to every consultation.}

The new GP contract has acted as a catalyst for engagement with the technology because payment for work done is by results and needs to be evidence-based. The PCT has online access into GP systems and can download data at will. It is no longer sufficient to leave the practice administrative staff to do the data entry as clinical data must be collected at each consultation.

This has led to a number of issues impacting on the practices. Training of all staff in the use of the systems is essential. Doctors need to understand the finer points of clinical coding to ensure that the correct data is captured at the source. This has been difficult for some. Nevertheless, if done well, it can provide a great deal of data for evidence-based medicine and may contribute to the future health of the population, something which the government insists on happening. The nurse practitioners (higher grade nurses who have the authority to perform delegated diagnostic work and prescribe certain drugs) have been instrumental in developing protocols for collection of clinical data on EMIS and the management of patient treatment for long-term conditions such as diabetes. This has caused some growing tensions in the practices where they are based as their new roles have yet to be clearly defined and the interface with GPs has the potential to be politically contested ground. 
Individual: From the perspective of the individual within the pilot practices, there are some major issues if the diffusion of ICT is to progress and be used innovatively. First, the practice managers generally felt that the onus was on them to deliver much of the data and this meant they relied on being up to date with all aspects of the EMIS system pertaining to their area. They have limited budgets for staff training and therefore undertook on-the-job training with all staff, including doctors, in their practice. Two comments from practice managers:

In the private sector there is a lot more training, development and coaching regarding change.

I've never had any formal training on the computer system.

This lack of training and resource was putting them under a lot of pressure at work and leading to feelings of being under valued. Although paid at a reasonable rate, they were unsure how the new GP contract payment system would work and suggested that it would generally only financially benefit the GPs.

The practice managers were also in the front line in terms of patient contact and, although not extensive, were increasingly using e-mail to communicate with patients. They saw this as beneficial to both practice and patient. However, the GPs did not want e-mail communication with patients and did not see any benefits to themselves. On the contrary, they felt this would add an unacceptable burden to workloads.

From the clinical practitioner perspective, ICT was impacting on them in different ways. One GP, who had been in practice for about 25 years, felt that the ICT was causing some difficulty and was becoming a barrier in the consultation room, leading to a poorer level of service to patients. She stated:

Consultation time is just 10 minutes, it's barely enough time to speak to someone without turning around and start working on the computer...I'm doing a 4 hour surgery in the morning and 4 hours in the afternoon, and absolutely brain dead at the end of it.

There was also a feeling among the GPs that GP medicine was becoming much more mundane and they were seeing less acute cases due to the way out-of-hours medicine was now being managed and the intervention of NHS direct. Also with the government's initiative on targeted interventions and possibly the role of the nurse practitioners, some GPs believed they could be deskilled. The nurse practitioners, however, were very upbeat about their emerging roles and saw themselves as advocates of ICT, one stating:

\section{I think we are taking on a lot of new roles, taking over a lot of things that} doctors would be doing.

Their nurse practitioner education program was underpinned by ICT and its use to support the patient consultation. Many used the decision support system Prodigy for advice on drug prescribing and drug conflicts when dealing with patients. The doctors 
interviewed did not use Prodigy. It was not on their horizon at all, even though it had been initially developed in the North East of England with GPs.

Environment: For the vast majority of those interviewed, the NHS national strategy on ICT had passed them by. Their horizon was the PCT and only those who were part of some external network had any idea that there was a national strategy. The practice managers generally met regularly at the PCT headquarters and were exposed only to relevant issues from the strategy. However, these were generally contextualized for the PCT. They did not see the big picture nor that they were part of a much larger initiative. An example of this was when we asked whether they were familiar with the current debate around local service providers (there are five clusters of LSPs in England, spread across geographical sectors and comprised of major consortia of consultancy companies and ICT vendors; Accenture heads the North East LSP), and that if the various contracts were signed they might lose EMIS, their current GP system, for a new one called Lorenzo (Accenture's preferred system). Only one interviewee was aware of this and the others were quite shocked that there had been no consultation on the decision, particularly when they were perfectly happy with EMIS.

Another initiative that was looming large on the government's agenda was "Choose and Book," where needy patients could, during a GP consultation, be referred through an ICT link to a hospital and consultant of their choice. None of the practices we visited had begun any form of discussion with local hospitals about how this would be implemented locally or even knew whether there was a system in place that could facilitate the process. This system was due to be implemented by December 2005 with current indications that the system may now not be ready for another year at the earliest.

Culture within organizations can often determine whether ICT diffusion will succeed. In the pilot practices studied, it was apparent that a culture change was emerging through a number of factors. A major factor was the introduction of new staff into the practices. Three of the practice managers had come from the private sector and were used to working in different ways. This "can do" approach was having a dramatic impact on how the surgery worked. Practices were working in more effective and efficient ways and tasks were getting done. The hierarchy within these practices is also changing and becoming much more team based with nurses working alongside the administrators to address patients' needs. Some doctors are aligning with this approach but other still need to maintain the status quo. ICT and the need for common shared goals such as standardized patient data are also facilitating this culture change but the evidence for this is only emerging. The difficulty for the PCTs will be keeping these champions of change and ICT diffusion and, on limited budgets, rewarding their efforts.

Organization: It is important for all concerned that innovations or ICT diffusion experience is communicated to share good practice and discuss future developments. To some extent, each of the three groups interviewed were part of networks but these networks were not ICT focused nor did they have ICT as an agenda item when formally constituted. The doctors met formally at the PCT to discuss clinical issues and new medical developments. They also were part of an informal network as many went to medical school together around the same time. What is obvious from our study is that GP practices are not all embracing ICT is the same way and the PCT was unable to engage any of the Luddites in our research. Even though the new GP contract expects GPs to work in certain ways, a number are not necessarily using EMIS to deliver 
requirements. How this will evolve and how the PCT will deal with transgressors is still to be determined.

The nurses do not appear to be well networked and only those who are studying in the nurse practitioners program have access to other similar individuals at a regional or national level. Thus they get to discuss more strategic issues but out of context to their local issues. In their practice they see themselves as part of the practice team and share information across the practice rather than across the PCT.

The practice managers are at the forefront of the ICT initiative and changes in the PCT. They meet regularly at the PCT but agendas are determined by the PCT management and generally relate to financial data and the new contract. The practice managers would like to network further to discuss their issues and share good practice and this may evolve through the efforts of new entrants into the arena. If practices are to further develop ICT and related systems, these individuals are the champions and the change agents. It will be through their efforts that diffusion will benefit the patients and bring about the evidence-based practice that the government believes is worthwhile.

Implications for organizational resilience: In relation to the work of Riolli and Savicki (2003), our research findings show that there are significant vulnerabilities and stresses related to the rapid and enforced diffusion of ICTs throughout the NHS and among staff in general medical practices. At the level of the individual, staff are coping by adopting processes of informal learning and modifying the technologies in use, such as the creation of templates by practice nurses. Formal and informal groups, such as GP committees within the PCT and also the practice managers' forums, are enabling sharing of good practice, ideas, advice, and dissemination of the official strategies and policies in a second-hand way - often making up for a lack of official communications. The organization as a whole, however, is not as agile in terms of responding to the innovations and changes required within the time scales stipulated by the government's political agenda. The net result is that the diffusion of ICT within general medical practices and across the PCT is problematic and slower than expected.

\section{DISCUSSION AND CONCLUSION}

This section explores some of the pertinent issues highlighted by this research project and reflects on the political process that influences the diffusion of ICT and modernized working practices in the NHS; the outcomes of which will determine the level of organizational resilience.

The macro politics of ICT adoption and diffusion: Our research has shown that in the PCT very few of the staff in the study were aware of either the political or technology driven agendas. They did not see themselves as part of a major ICT initiative nor as using innovative technology. They were generally unaware that their GP system, EMIS, was likely to be replaced by another due to contract negotiations at a national level. They were shocked that they had not been consulted. Another major government innovation, the Choose and Book scheme, whereby patients could be referred electronically for consultation to a hospital, had hardly been explored in the PCT. Yet this system was intended to be live across the UK by December 2005. Thus the government rhetoric and local reality of DOI are completely incongruent in this PCT. Patient choice 
is seen as a cornerstone of the strategy alongside a modern technologically driven health service. The government has used this message extensively to gain votes but at a cost, perhaps, of totally unrealistic expectations from the public.

Micro-politics: the impact of the NHS ICT strategy on GPs and their practices: As has already been stated above, the strategy per se has hardly entered the consciousness of the local GP practices. However, the new GP contract has had a much bigger impact and has brought ICT adoption and innovation, to a lesser extent, to the fore. The reason for this is the requirement to collect management information at the source to ensure funding for primary care at the GP level. However, some aspects of ICT innovations that have been assimilated into the normal working life of many organizations such as e-mail, electronic booking systems, and mobile computing are being resisted by GPs in the pilot study.

It is evident that many individual and task related factors are influencing the diffusion process. User resistance is not overt in many cases, but just manifested through lack of engagement with the new technologies and protectionism due to maintaining traditional professional boundaries. Much learning is taking place on an individual basis with the importance of peer to peer training underestimated. The national agenda for modernization is being pushed through the organization by the strict imposition of quality indices, targets, and monitoring. The lure of financial rewards for doctors and increased esteem through enhanced professional status of nurse practitioners are significant factors. ICT plays a vital role in achieving targets and supporting the decision making processes of less qualified health professionals.

The contribution to IS theory and the research approach adopted: From our perspective, this was the first detailed study that used a DOI research framework to analyze complex political processes within small organizations in healthcare. This contributes to our understanding of IT adoption within professionally dominated cultures. It also presents an alternative or complimentary approach to other organizational research in IS such as actor network theory (Latour 1991; Munir and Jones 2004), structuration theory (Jones 1999; Walsham 2002) and soft systems methodology (Checkland 1981).

The use of semi-structured interviews to gather data from three key perspectives within general medical practices provided a rich insight into the actual politics of ICT adoption and diffusion. The independence of the researchers was critical to the success of this exercise alongside the protection of anonymity for the respondents. The template methodology was also successful in determining key categories and codes around which to base both the interviews and also the initial data analysis. The literature on diffusion of innovation research, however, provided a different and alternative avenue upon which to examine and analyze the data. In particular, the adapted framework extracted from Mustonen-Ollila and Lyytinnen (2003) enabled a more sophisticated understanding of the data and provided a new structure from which to explain the politics of the diffusion process. This was more congruent with Baskerville and Pries-Heje's (2001) view that complex political processes associated with DOI studies may be better understood by adopting an interactive and ecological model of diffusion. There are limitations to our study, however. This is mainly due to it being on a small scale across five practices within one PCT organization, and also due to the self selecting bias as a result of having to solicit volunteers as opposed to selecting a more representative sample. 


\section{References}

Baskerville, R., and Pries-Heje, J. "A Multiple-Theory Analysis of a Diffusion of Information Technology Case," Information Systems Journal (11), 2001, pp. 181-212.

Benson, T "Why General Practitioners Use Computers and Hospital Doctors Do Not-Part 1: Incentives, British Journal of Medicine (325), November 9, 2002, pp. 1086-1089.

Bloomfield, B. P., Coombs R., Owen, J., and Taylor, P. "Doctors as Managers: Constructing Systems and Users," in B. Bloomfield, R. Coombs, D. Knights, and Littler (eds.), The National Health Service in IT and Organizations, Oxford, UK: Oxford University Press, 1997, pp. 112- 132.

Caldeira, M. M., and Ward, J. M. "Using Resource-Based Theory to Interpret the Successful Adoption and Use of Information Systems and Technology in Manufacturing Small and Medium Sized Enterprises," European Journal of Information Systems (12), 2003, pp. 127-141.

Checkland, P. Systems Thinking, Systems Practice, Chichester, UK: Wiley, 1981.

Clarke C. L., and Wilcockson, J. "Professional and Organizational Learning: Analyzing the Relationship with the Development of Practice,"Journal of Advanced Nursing (34:2), 2002, pp. 264-272.

Clarke C. L., and Wilcockson J. "Seeing Need and Developing Care: Exploring Knowledge for and from Practice," International Journal of Nursing Studies (39:4), 2001, pp. 397-406.

Cragg, P. B. "Benchmarking Information Technology Practices in Small Firms," European Journal of Information Systems (11), 2002, pp. 267-282.

DOH. "Delivering $21^{\text {st }}$ Century IT Support for the NHS," Department of Health National Strategic Program, 2005 (available online through http://www.connectingforhealth.nhs.uk/ publications/).

Drucker, P. "The Discipline of Innovation," Harvard Business Review, May-June 1985, pp. $67-72$.

Doherty, N. F., Coombs, C., and Loan-Clarke, J. "Exploring the Duality of Information Technology in Community Health Trusts," Research Series Working Paper, Business School, Loughborough University, 2004.

Doherty, N. F., King, M., and Marples, C. G. "The Impact of Hospital Information Support Systems on the Operation and Performance of Hospitals," Information Systems Review (1:1), 2000, pp. 97-107.

Grandon, E. E., and Pearson, M. J. "Electronic Commerce Adoption: An Empirical Study of Small and Medium US Businesses," Information and Management (42), 2004, pp. 197-216.

Horne, J. F., and Orr, J. E "Assessing Behaviors that Create Resilient Organizations," Employment Relations Today (24), 1998, pp. 29-39.

Jones, M. "Structuration Theory," in W. Currie and R. D. Galliers (eds.), Rethinking Management Information Systems: An Interdisciplinary Perspective, Oxford, UK: Oxford University Press, 1999.

Kautz, K., and Larsen, E. A. "Diffusion Theory and Practice: Disseminating Quality Management and Software Process Innovations," Information Technology and People (13:1), 2000, pp. 11-26.

Kim, C., and Galliers, R. D. "Toward a Diffusion Model for Internet Systems," Internet Research (14:2), 2004, pp. 155-166.

King, N. "Using Templates in Thematic Analysis of Text," in C. Cassell and G. Symon (eds.), Essential Guide to Qualitative Methods in Organizational Research, London: Sage Publications, 2004. 
Latour, B. "Technology is Society Made Durablem" in J. Law (ed.), A Sociology of Monsters: Essays on Power, Technology and Domination, London: Routledge, 1991, pp. 103-131.

Munir, K. A., and Jones, M. "Discontinuity and After: The Social Dynamics of Technology Evolution and Dominance," Organization Studies (25:4), 2004, pp. 561-581.

Mustonen-Ollila, E., and Lyytinen, K. "Why Organizations Adopt Information System Process Innovations: A Longitudinal Study Using Diffusion of Innovation Theory, Information Systems Journal (13), 2003, pp. 275-297.

Newton, J., Graham, J., McLoughlin, K., and Moore, A. "Receptivity to Change in a General Medical Practice," British Journal of Management (14), 2003, pp. 143-153.

Packwood, T., Pollit. C., and Roberts, S. "Good Medicine? A Case Study of Business Process Re-engineering in a Hospital," Policy and Politics (26:4), 1998, pp. 401-415.

Pettigrew, A., Ferlie, E., and McKee, L. Shaping Strategic Change, London: Sage Publications, 1994.

Poon, S., and Swatman, M. C. "An Exploratory Study of Small Business Internet Commerce Issues, Information and Management (35), 1999, pp. 9-18.

Riolli, L., and Savicki, V. "Information System Organizational Resilience,” Omega (31), 2003, pp. 227-223.

Rogers, E. M. Diffusion of Innovations ( $4^{\text {th }}$ ed.), New York: The Free Press, 1995.

Schroeder, R. G., Van de Ven, A. H., Scudder, G. D., and Polley, D. "The Development of Innovation Ideas," in A. H. Van de Ven, H. L. Angle, and M. S. Scott Poole (eds.), Research on the Management If innovations: The Minnesota Studies, New York: harper and Row, 1989, pp. 107-134.

Southern, A., and Tilley, F. "Small Firms and Information and Communication Technologies (ICTs): Toward a Typology of ICTs Usage," New Technology Work and Employment (15:2), 2000, pp. 138-153.

Wainwright, D., and Waring, T. "The Information Management and Technology Strategy of the UK National Health Service: Determining Progress in the NHS Acute Hospital Sector," The International Journal of Public Sector Management (13:3), 2000, pp. 242-259.

Walsham, G. "Cross Cultural Software Production and Use: A Structurational Analysis," MIS Quarterly (26:4), 2002, pp. 359-380.

Waring, T., and Wainwright, D. "Communicating the Complexity of Computer-Integrated Operations: An Innovative Use of Process Modeling in a North East Hospital Trust," International Journal of Operations and Production Management (22:4), Special Issue on Healthcare Systems, 2002, pp. 394-411.

\section{About the Authors}

David Wainwright is a reader in Information Systems and Head of Collaborative Research at the School of Computing, Engineering and Information Sciences, Northumbria University, Newcastle upon Tyne. He has a Ph.D. in Information Systems and his main area of research focuses on developing organizational approaches to information systems integration and implementation. David has extensive high level experience of consulting with large and small organizations, both in commercial businesses and also the healthcare sector. David has an extensive portfolio of internationally refereed articles and has published in many major journals. He has been invited as keynote speaker at many international conferences. He received a highly commended award for his paper in the International Journal of Operations and Production Management. David is a member of the British Computer Society and also serves as an elected board member of the UK Academy for Information Systems (UKAIS). David was the organizing and program chair for the UKAIS 2005 conference. He can be reached at david.wainwright@ unn.ac.uk. 
Teresa Waring is Associate Dean of Business and Management at Sunderland University Business School and is Director of Research for the subject area. Her research is in the area of critical information systems with a focus on systems integration. She has a Ph.D. and an M.Sc. in computer-based information systems and her main research area of interest is the implementation of information systems. Although Teresa has spent most of her career in education, she has acted as a consultant to a large number of organizations both in the private and public sectors and retains close links with many of them. She has presented research papers at many conferences, both in the UK and Europe, and has a number of internationally refereed articles. In 2002, she won the best paper award at the Business Innovation in the Knowledge Economy Conference at IBM Warwick, UK, and was highly commended for her paper in the International Journal of Operations and Production Management. She can be reached at teresa.waring@ sunderland.ac.uk. 